\title{
Rapidly rotating neutron star progenitors
}

\author{
K.A. Postnov ${ }^{1,2,3 \star}$, A.G. Kuranov ${ }^{1}$, D.A. Kolesnikov ${ }^{1,2}$, S.B. Popov ${ }^{1}$, N.K. Porayko ${ }^{1,2,3,4}$ \\ ${ }^{1}$ Sternberg Astronomical Institute, Moscow M.V. Lomonosov State University, Universitetskij pr., 13, Moscow 119992, Russia \\ ${ }^{2}$ Faculty of Physics, M.V. Lomonosov Moscow State University, Leninskie Gory, Moscow 119991, Russia \\ ${ }^{3}$ Institute of Theoretical and Experimental Physics, Moscow, Russia \\ ${ }^{4}$ Max-Planck-Institut für Radioastronomie, Bonn, Germany
}

Received ... Accepted ...

\begin{abstract}
Rotating proto-neutron stars can be important sources of gravitational waves to be searched for by present-day and future interferometric detectors. It was demonstrated by Imshennik that in extreme cases the rapid rotation of a collapsing stellar core may lead to fission and formation of a binary proto-neutron star which subsequently merges due to gravitational wave emission. In the present paper, we show that such dynamically unstable collapsing stellar cores may be the product of a former merger process of two stellar cores in a common envelope. We applied population synthesis calculations to assess the expected fraction of such rapidly rotating stellar cores which may lead to fission and formation of a pair of proto-neutron stars. We have used the BSE population synthesis code supplemented with a new treatment of stellar core rotation during the evolution via effective core-envelope coupling, characterized by the coupling time, $\tau_{c}$. The validity of this approach is checked by direct MESA calculations of the evolution of a rotating $15 M_{\odot}$ star. From comparison of the calculated spin distribution of young neutron stars with the observed one, reported by Popov and Turolla, we infer the value $\tau_{c} \simeq 5 \times 10^{5}$ years. We show that merging of stellar cores in common envelopes can lead to collapses with dynamically unstable proto-neutron stars, with their formation rate being $\sim 0.1-1 \%$ of the total core collapses, depending on the common envelope efficiency.
\end{abstract}

Key words: gravitational waves, stars: neutron, stars:rotation

\section{INTRODUCTION}

The present-day attempts to model the explosion of core collapse supernova $(\mathrm{SN})$ in the framework of neutrino mechanism proposed half a century ago (Colgate \& White 1966) are almost successful in 3D (see, e.g., recent calculations Melson et al. 2015 and references therein). Clearly, rotating neutron stars (NS) that result from corecollapse $\mathrm{SNe}$ and are observed as radio pulsars shows that the rotation of the collapsing core of a massive star should be taken into account in the SN explosion calculations (see Langer 2012 for a review of rotational effects on the evolution of massive stars in single and binary systems). The rotation and magnetic fields in stellar cores can be so important that an alternative SN explosion mechanism, called magneto-rotational explosion, was suggested soon after the neutrino mechanism was proposed (Bisnovatyi-Kogan 1971; LeBlanc \& Wilson 1970) (see, e.g., Ardeljan et al. 2005 for results of 2D MHD simulations). Recent 3D MHD numerical simulations (e.g. Mösta et al. 2015) suggest that rapidly rotating cores of massive stars with magnetic field may trigger the most energetic astrophysical explosions including long gamma-ray bursts and super-

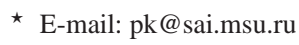

luminous supernovae. Non-axisymmetric instabilities of rapidly rotating proto-NSs may be interesting sources of astrophysical gravitational waves (see a summary of recent studies and prospect for future advanced LIGO-VIRGO searches in Gossan et al. 2016; Abbott et al. 2016). GWs (gravitational waves) are also promising source of the direct probe of rapid rotation in nucleus of core collapse supernovae through observation of circular polarization of GW signal from possible hydrodynamic instabilities at the postbounce phase (Hayama et al. 2016).

In extreme cases, the fast rotation of the iron-oxygen core of a massive star prior to collapse can lead to the formation of dynamically unstable proto-NSs (Centrella et al. 2001; Baiotti et al. 2007). For instance, in classical incompressible Maclaurin spheroid approximation (Chandrasekhar 1969; Durisen \& Tohline 1985), when the ratio of the rotational energy $T$ to gravitational energy $W$, $\beta=T /|W|>\beta_{d y n}$ achieves the critical value $\beta_{d y n} \simeq 0.27^{1}$, the barmode instabilities are formed and can consequently lead to fission

\footnotetext{
1 The analysis in full GR shows (Baiotti et al. 2007) that the classical result for incompressible Maclaurin spheroids remains valid, with $\beta_{d y n} \sim 0.25-$ 0.27 ; here we will use the classical value $\beta_{d y n}=0.27$.
} 


\section{Postnov et al.}

of the proto-NS into two parts. As a result, a close binary NS may be formed during the core collapse. The orbital evolution driven by GW emission brings the lightest (having higher radius) component into contact with Roche lobe leading to the mass transfer from the lightest onto the more massive component. When the mass of the lightest companion decreases down to a minimum possible mass of stable NS, $\sim 0.1 M_{\odot}$, density of the matter (and hence Fermimomentum of electrons) becomes too low and enables neutrons in neutron-rich nuclei to beta-decay in a fraction of a millisecond, which ends up with an explosion of the $\sim 0.1 M_{\odot}$ NS remnant producing a powerful neutrino outburst. This scenario was proposed by Imshennik (1992) to explain unusual neutrino signals from SN 1987A; (see Imshennik \& Nadyozhin 1992, Imshennik 2011 for a review and references). The approximate analytic solution of the the GW signal (waveform) emitted by these types of the sources was found in (Imshennik \& Popov 1998; Imshennik 2008). During the first stage of merging, the amplitude and frequency of the GW signal increases, achieving the maximum value in the beginning of the second stage, when the low-mass component fills its Roche Lobe.

The idea of rapidly rotating collapsing core fragmentation into two pieces has several important astrophysical implications. For example, this can lead to high kick velocities of a NS or a black hole resulting from the ultimate merging of two NSs (Colpi \& Wasserman 2002), it may be important in some long gamma-ray burst progenitor models (Lipunova et al. 2009) and can be additional source of GW signals from merging binary NS system (Imshennik \& Popov 1998; Davies et al. 2002). The latter is especially important for the ongoing searches of GW by the presentday advanced GW detectors (Aasi et al. 2015; Acernese et al. 2015; Abbott et al. 2016). From the point of view of GW detection, the precise form of the GW signal and astrophysical rate of events is of major importance (Sathyaprakash \& Schutz 2009). While the former is very well studied for coalescing binary NSs (e.g. Blanchet 2014), the event rate of double NS coalescences suffers from many astrophysical uncertainties and model assumptions (Voss \& Tauris 2003; Postnov \& Yungelson 2014; de Mink \& Belczynski 2015).

The main motivation of the present study is to calculate, by means of the population synthesis method, the expected formation rate of rapidly rotating cores of massive stars with parameter $\beta$ high enough that can potentially lead to noticeable astrophysical manifestations. We will focus only on the angular momentum of the core and will not consider magnetic fields, which are prerequisite for magneto-rotational and magnetar-powered supernova explosions and long GRBs. To this end, we will treat the rotation of the stellar core in single and binary massive stars using the effective coupling between the core and envelope of the star (Allain 1998). We check the applicability of such a simplified description to the stellar core rotation by comparing the results with calculations using MESA evolutionary code (Paxton et al. 2011). From comparison of the calculated initial NS rotational periods with the distribution derived from pulsar observations, we find the effective coupling constant between the core and envelope (coupling time $\sim 5 \times 10^{5}$ years). With this coupling, we calculate the distribution of iron core rotation (the $\beta$-parameter) in single and binary stars. In single stars, the rotation of the core is too slow for strong rotational instabilities to develop. In binary systems, the rapid rotation arises at stages of coalescence of non-degenerate stellar cores during the common envelope stage. In our calculations we take into account tidal synchronization of the stellar envelope in close binaries and loss of stellar envelope's angular momentum by stellar wind, as implemented in the BSE population synthesis code (Hurley et al. 2000, 2002). This is a novel element compared to some previous population synthesis studies, which assumed rigid rotation of the entire star in a close binary system (e.g. Bogomazov \& Popov 2009; Lipunova et al. 2009). We find that for the standard parameters of close binary evolution the fraction of rapidly rotating cores can reach $0.1-1 \%$ of the total number of core collapses. This rate is comparable to an optimistic Galactic rate of double NS coalescences of about $10^{-4}$ per year (Abadie et al. 2010; Postnov \& Yungelson 2014).

The structure of the paper is as follows. In Section 2 we briefly describe the stellar core rotation and its implementation into the modified BSE code. In Section 3 we discuss the initial rotation periods of NSs as derived from pulsar studies. In Section 4 we presents the results of our calculations, and Section 5 gives summary of the present study. In Appendix A we compare the results of core rotation calculation of a $15 M_{\odot}$ star with detailed calculation by the MESA code. Appendix B presents the typical evolutionary tracks of binary stars that lead to rapidly rotating stellar cores prior to collapse.

\section{DESCRIPTION OF THE STELLAR CORE ROTATION}

Stellar rotation is a very broad and topical field of astrophysical studies and is recognized to be a key factor, in addition to the mass and chemical composition, determining stellar evolution (Maeder \& Meynet 2000). The core-envelope coupling can be due to magnetic torques (e.g. Spruit 2002; Braithwaite \& Spruit 2015) or internal gravity waves excited by convective motions (Zahn et al. 1997).

In the present study, the rotational evolution of the stellar core is effectively described as follows. Consider a star consisting of core and envelope with moment of inertia $I_{c}$ and $I_{e}$, respectively. The total angular momentum of the star is $J=I_{c} \omega_{c}+I_{e} \omega_{e}$, where $\omega_{c}$ and $\omega_{e}$ are core's and envelope's angular velocity, respectively, assuming rigid rotation. Following Allain (1998), we assume that the stellar core with moment of inertia $I_{c}$ is effectively coupled with the stellar envelope with moment of inertia $I_{e}$ (e.g. due to magnetic torques (e.g. Spruit 2002; Braithwaite \& Spruit 2015) or internal gravity waves excited by convective motions (Zahn et al. 1997)) as follows:

$$
\frac{d J_{c}}{d t}=-\frac{I_{c} I_{e}}{I_{c}+I_{e}} \frac{\omega_{c}-\omega_{e}}{\tau_{c}}
$$

where $J_{c}$ is the angular momentum of the core and $\tau_{c}$ is the coreenvelope coupling time. If the latter is too long (e.g., comparable to the characteristic time of stellar evolution), the stellar core essentially evolves conserving its own angular momentum.

The initial rotational periods of main-sequence stars were adopted as in the BSE (Binary Star Evolution) code (Hurley et al. 2000 ) which uniquely relates the equatorial rotational velocity of a zero-age main-sequence star with its mass, $v_{\text {rot }}(M)$ (see Eq. (107) in that paper). Initially, the star is assumed to rotate rigidly, $\omega_{c}=\omega_{e}$. During evolution, the core and envelope rotation changes due to variation of the core and envelope moment of inertia, tidal and stellar wind torques applied to the envelope as implemented in the original BSE code, and the core-envelope coupling discussed above. However, we find that that the initial rotation does not affect the fraction of rapidly pre-collapse stellar cores, which is the main purpose of our study.

The comparison of our effective description of the stellar core 

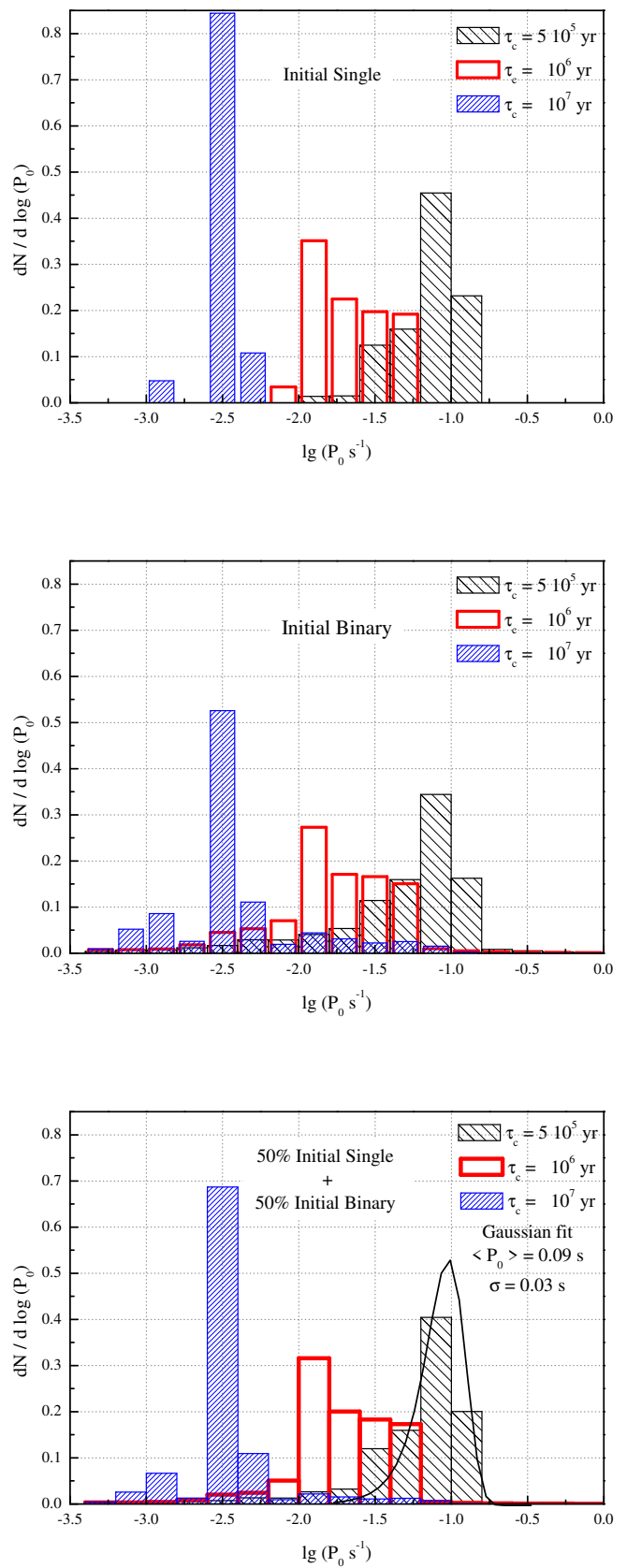

Figure 1. Initial spin periods of NSs originated from single, binary and equal number of single and binary progenitors (upper, middle and bottom panel, respectively), for different values of the core-envelope coupling time $\tau_{c}=5 \times 10^{5}, 10^{6}$ and $10^{7}$ years. The solid line in the bottom panel shows the best-fit Gaussian for the calculated $d N / d P_{0}$ distribution with parameters shown in the inset.

rotation with more detailed calculations by MESA code are presented in the Appendix A.

To quantify the core rotation before the collapse, we will use the ratio of the rotational energy to the gravitational potential energy of the core, $\beta=T /|W|$. The kinetic energy of a rigidly rotating core is

$$
T=\frac{k M_{c} R_{c}^{2} \omega^{2}}{2},
$$

where $M_{c}$ and $R_{c}$ is the mass and radius of the core, respectively, and $k$ is the numerical factor depending on the density distribution. For example, for polytropic structure with $n=3 / 2, k \approx 0.21$, and for $n=3, k \approx 0.075$. The stellar core before collapse is be described by a polytropic configuration with index $n=3$, and the gravitational potential energy for such a core is

$$
W=-\frac{3}{5-n} \frac{G M_{c}^{2}}{R_{c}},
$$

where $G$ is the Newton gravitational constant.

\section{INITIAL NEUTRON STAR SPIN PERIODS}

In the present study, we will compare the initial period distributions of NSs calculated by the BSE code for different values of the core-envelope coupling parameter $\tau_{c}$ with the initial NS spin period distribution $d N / d P_{0}$ as inferred from observations. The latter requires a separate discussion.

From the observational point of view, there are several populations of young isolated NSs: radio pulsars, magnetars (soft gammaray repeaters and anomalous X-ray pulsars), central compact objects (CCOs) in SNRs, rotating radio transients (RRATs), and socalled X-ray dim isolated NS, aka the 'Magnificent seven'. Despite the fact that all of these sources are relatively young NSs and in many cases rough age estimates can be obtained, it is very difficult to estimate the initial spin distribution for the majority of them. There are three main difficulties:

- It is necessary to have a precise model of the NS spin-down;

- It is necessary to know how several key parameters evolve (magnetic field and magnetic inclination angle);

- It is necessary to have precise (better than a few 10\%) independent age estimate.

Significant evolution of the magnetic field can be expected in magnetars. Therefore in this case the determination of $P_{0}$ is the most difficult task. Similar conclusions can be made about the Magnificent seven, as they also represent evolved NSs with initially large magnetic field (Popov et al. 2010). Thus, CCOs and radio pulsars (RRATs can be considered together with radio pulsars) seem to be the most appropriate objects for estimates of the initial NS spin periods.

In a few cases where NSs are related to historical SNe, the NS age is known rather accurately (e.g. Clark \& Stephenson 1982; Imshennik 2000). However, other estimates are required to get a larger statistics. There are two main approaches to obtain independent age estimates for tens of NSs. The less precise one is from kinematic considerations. The so-called kinematic age have been used by Noutsos et al. (2013) to derive $P_{0}$ for several tens of radio pulsars. The second, more precise approach is based on the NS age estimation from the SNR observations. This method has been used by many authors. For example, in the recent attempt Popov \& Turolla (2012) about 30 NS+SNR pairs were analyzed.

In addition, population synthesis modeling have also been used to probe the initial NS spin period distribution (see, e.g. Faucher-Giguère \& Kaspi 2006; Popov et al. 2010; Gullón et al. 2014, 2015).

In brief, all studies (using samples of sources with known ages 
and population studies) demonstrate that radio pulsars (which dominate the samples) can have relatively broad initial spin period distribution up to $0.5-1$ second, and typical periods are about $100 \mathrm{~ms}$.

Popov \& Turolla (2012) did not try to produce an exact distribution, as the available statistics of objects is not large enough. But it was possible to probe different popular analytical distribution vs. cumulative initial spin period distribution derived from PSR+SNR pairs. The authors demonstrated that both very broad flat and very narrow ( $\delta$-function like) distributions do not fit well the data. On the other hand, Gaussian distributions with $\left\langle P_{0}\right\rangle \sim 0.1-0.2 \mathrm{~s}$ and $\sigma \sim 0.1-0.2 \mathrm{~s}$ are found to be in agreement with the data.

Noutsos et al. (2013) produced the initial spin period distribution using several tens of radio pulsars with known kinematic ages. Their distribution is bimodal. One mode is in correspondence with findings by Popov \& Turolla (2012). In addition, they proposed that there is a "tail" of young NSs with initial spin periods $P_{0} \sim 0.5$ $0.7 \mathrm{~s}$. However, the latter feature can be a result of some evolutionary effects (magnetic field decay or emergence) which have not been taken into account in that study (see Igoshev \& Popov 2013).

In population synthesis studies, different authors infer the NS initial spin period distribution from fitting the present-day radio pulsar properties by model distributions. This approach is based on many assumptions, and it is difficult to derive an independent exact form and parameters of the $P_{0}$ distribution. For example, the possibility of "injection" of pulsars at spin periods $\sim 0.5 \mathrm{~s}$ cannot be rejected (Vivekanand \& Narayan 1981; Vranesevic et al. 2004). Generally, results of different studies are not in contradiction with analysis of samples of young NSs with known ages. Initial spin periods $\sim 100 \mathrm{~ms}$ are favored, but the distributions are not very narrow, and allow some pulsars to be born with ms periods, and some - with $P_{0} \sim 0.5 \mathrm{~s}$.

Theoretical estimates of the $P_{0}$ distribution remain rather uncertain (see recent reviews and references in Miller \& Miller 2015; Fuller et al. 2015). Several mechanisms can significantly affect the initial NS rotation. In particular, the standing accretion shock instability (SASI) can modify the NS spin (Guilet \& Fernández 2014 and references therein). Effect of fall-back accretion onto the nascent NS can also be important (Menou et al. 2001).

Therefore, in the present study we will assume, guided by observations, that initial NSs periods have a Gaussian-like distribution centered on $\sim 100 \mathrm{~ms}$ with a comparable dispersion.

\section{RESULTS}

\subsection{Core-envelope coupling time}

First, we performed BSE population synthesis calculations of the initial NS spin periods from single and binary progenitors. A NS was assumed to be formed in the ZAMS mass range from $M_{\min }=8 M_{\odot}$ to $M_{\max }=35 M_{\odot}$. The rotation of the stellar He and $\mathrm{CO}$-cores was treated as described above. The mass of the pre-collapse iron-oxygen core was assumed to be $1.8 M_{\odot}$, and its rotation was calculated by assuming angular momentum conservation after the CO-core stage. In other terms, a mass of $1.8 M_{\odot}$ was cut from the CO-core described by $n=2$ polytrope (which is a reasonable approximation at the beginning of the CO-stage) with the angular momentum $J_{1.8}=I_{1.8} \omega_{C O}$, where $I_{1.8}$ is the moment of inertia of the central $1.8 M_{\odot}$ part of the CO-core and $\omega_{C O}$ is the angular velocity of the $\mathrm{CO}$-core at this stage. Then the rotation of the iron-oxygen core was calculated assuming the $1.8 M_{\odot}$ $n=2$ rigidly rotating polytrope contracting to $n=3$ rigidly rotating polytrope (which is good approximation of the core sptructure prior to collapse) with the same mass and angular momentum, i.e. $\omega_{F e}=\omega_{C O} I_{1.8}(n=2) / I_{1.8}(n=3)$.

During the core collapse, the angular momentum conservation was assumed, $I_{1.8} \omega_{F e}=I_{N S} \omega_{N S}$, where the NS moment of inertia $I_{N S}=10^{45} \mathrm{~g} \mathrm{~cm}^{2}$. Therefore, the initial NS period was found as $P_{0}=\left(2 \pi / \omega_{N S}\right)$. (At this stage we neglect possible dynamical instabilities of the rapidly rotating stellar cores and young NSs.) The binary progenitors include all binary systems in which the initial primary's mass $M_{\max } \geqslant M_{1} \geqslant M_{\min }$. The standard binary population synthesis initial parameters were adopted: a flat distribution of the binary mass ratio $q=M_{2} / M_{1} \leqslant 1: d N / d q \sim q^{0}$; a flat distribution of the initial binary separation, $d N / d \log a=$ const and the distribution of initial binary periods by massive binaries proposed by Sana et al. (2012), $d N /(d \log P)^{\pi}=$ const with $\pi=-0.55$; alpha-description of the common envelope (CE) stage with the efficiency parameter $\alpha_{C E}$ (see, e.g. Postnov \& Yungelson 2014 for a detailed description of binary evolution and population synthesis parameters). Note that the natal kick velocity imparted to NS in core collapse is not important for our calculations as long as central kicks are considered that do not affect the NS spin (see, however, Spruit \& Phinney 1998 for alternative explanation of NS spins, which we do not consider here).

The resulted (normalized) distribution of NS initial periods $P_{0}$ is shown in Fig. 1 for a population of single, binary and equal fractions of single and binary progenitors (the upper, middle and bottom panel of Fig. 1, respectively) for different values of the coreenvelope coupling time $\tau_{c}=5 \times 10^{5}, 10^{6}$ and $10^{7}$ years. For each set of parameters, evolution of a population of $5 \times 10^{5}$ systems was calculated.

It is seen that the long (comparable to the stellar evolution) core-envelope coupling time essentially leads to the core evolution with angular momentum conservation and overproduction of rapidly rotating NSs. The evolution in binary systems (mostly via tidal interaction in close binaries) leads to wider $P_{0}$ distributions, but still essentially peaked at the same values as from single progenitors (the middle panel of Fig. 1), and therefore the initial $P_{0}$ distribution from a population of equal number of single and binary progenitors (the bottom panel of Fig. 1) does not significantly differ from that resulted from binary progenitors. In the bottom panel of Fig. 1, the calculated $d N / d P_{0}$ distribution for $\tau_{c}=5 \times 10^{5}$ years can be fitted by a Gaussian with $\left\langle P_{0}\right\rangle=0.09 \mathrm{~s}$ and $\sigma=0.03 \mathrm{~s}$ (the solid line), which is reasonably close to the distribution derived from observations (see Section 3). Thus we conclude that the coreenvelope coupling time should be close to $5 \times 10^{5}$ years to reproduce the initial NS period distribution. Note that further decrease in $\tau_{c}$ would shift the $P_{0}$ peak to longer periods, in contradiction with observations. Physically, a shorter $\tau_{c}$ means more effective coupling between the core and envelope, and the expansion of the envelope during the stellar evolution strongly spins down the stellar core. Below we shall take $\tau_{c}=5 \times 10^{5}$ years.

\subsection{On the magnetar formation from rapidly rotating cores}

Rapidly rotating NS have been frequently invoked as possible progenitors of magnetars, - i.e. highly magnetized NSs, - due to an effective dynamo mechanism, enhancing the magnetic field in a proto-NS (Thompson \& Duncan 1993; see Turolla et al. 2015 for a recent review). It is unclear, however, if indeed a dynamo mechanism is responsible for generation of inferred high magnetic fields of magnetars, and if yes, which kind of dynamo is in operation. Therefore, it is difficult to specify exact initial conditions for a NS to become a magnetar, in particular, what should be the critical spin 
period of the proto-NS. For example, Thompson \& Duncan (1993) advocated the range $\lesssim 3 \mathrm{~ms}$, but this value can vary in different dynamo models.

Spin-up of the stellar core in magnetar progenitors due to binary evolution (accretion of angular momentum, tidal synchronization, coalescence) have been discussed by Popov \& Prokhorov (2006). There is observational evidence that magnetars SGR $1900+14$ and CXOU J1647-45 may have been born in binary systems. The first of them was studied by Davies et al. (2009). Analysis of the cluster in which SGR 1900+14 was born brought the authors to the conclusion that the source progenitor was likely a member of a binary, since otherwise it would be difficult to bring in correspondence relative low progenitor mass $\left(17 \pm 1 M_{\odot}\right)$ with standard scenarios of stellar evolution and magnetar formation. The progenitor of the second source was studied by Muno et al. (2006) and recently in more details by Clark et al. (2014). In the last paper, the authors provided direct arguments in favour of the magnetar formation in a binary system and concluded that 'binarity is a key ingredient in the formation of at least a subset of magnetars'.

The fraction of magnetars among newborn NSs is estimated to be $\sim 10 \%$ (see, for example, Turolla et al. 2015 and references therein). The initial spin distributions of NS presented in Fig. 1 suggest that for the effective core-envelope coupling time $\tau_{c}<10^{6}$ yrs the fraction of rapidly rotating NSs (with initial spin periods $P_{0} \lesssim 10 \mathrm{~ms}$ ) is much smaller than $10 \%$, while $\tau_{c}>10^{6}$ yrs is in contradiction with isolated NS data. Therefore, we conclude that in the framework of our model description of the rotation of stellar cores the whole magnetar population cannot originate only from rapidly rotating proto-NSs.

\subsection{Stellar core rotation before the collapse}

In Fig. 2 we show the distribution of the rotation parameter $\beta$ of the core before the collapse for single NS progenitors (the upper panel) and for binary progenitors (the bottom panel) with $\tau_{c}=5 \times 10^{5}$ years. It is seen that the value of $\beta$ for single stars is always too small to allow the dynamical instability to develop. However, for binary progenitors the situation is much more interesting. Indeed, we see that there is a tail of rapidly rotating cores with high $\beta$. For the dynamical instability to develop, proto-NS should have $\beta>\beta_{d y n} \sim 0.27$. Since during the collapse with angular momentum conservation (by assumption) $\beta$ increases proportionally to the inverse radius of the contracting configuration, potentially interesting for the dynamical instability will be the cores with $\beta>\beta_{c r} \sim 2.7 \times 10^{-3}$, where we have conservatively assumed that the radius of a proto NS is 100 times smaller than that of the precollapse iron core ${ }^{2}$. The bottom panel of Fig. 2 shows that there can be a non-negligible fraction of such rapidly rotating stellar cores for the assumed $\tau_{c}=5 \times 10^{5}$ years. It is interesting to see what are the progenitor binary systems of such rapidly rotating cores.

From the present population synthesis calculations we find that all such rapidly rotating cores resulted from merging in the common envelope stage. Typical evolutionary tracks leading to such merging are listed in Tables B1-B5 in Appendix B. The eclipsing binary system GU Mon with early type companions of equal masses which are in contact can be an example of the binary system at the stage of the beginning of the common enve-

2 For example, in detailed calculations by Imshennik \& Molokanov (2009) the collapsing $1.8 M_{\odot}$ rapidly rotating iron-oxygen core described by $n=3$ polytrope had a radius of $3.75 \times 10^{8} \mathrm{~cm}$.
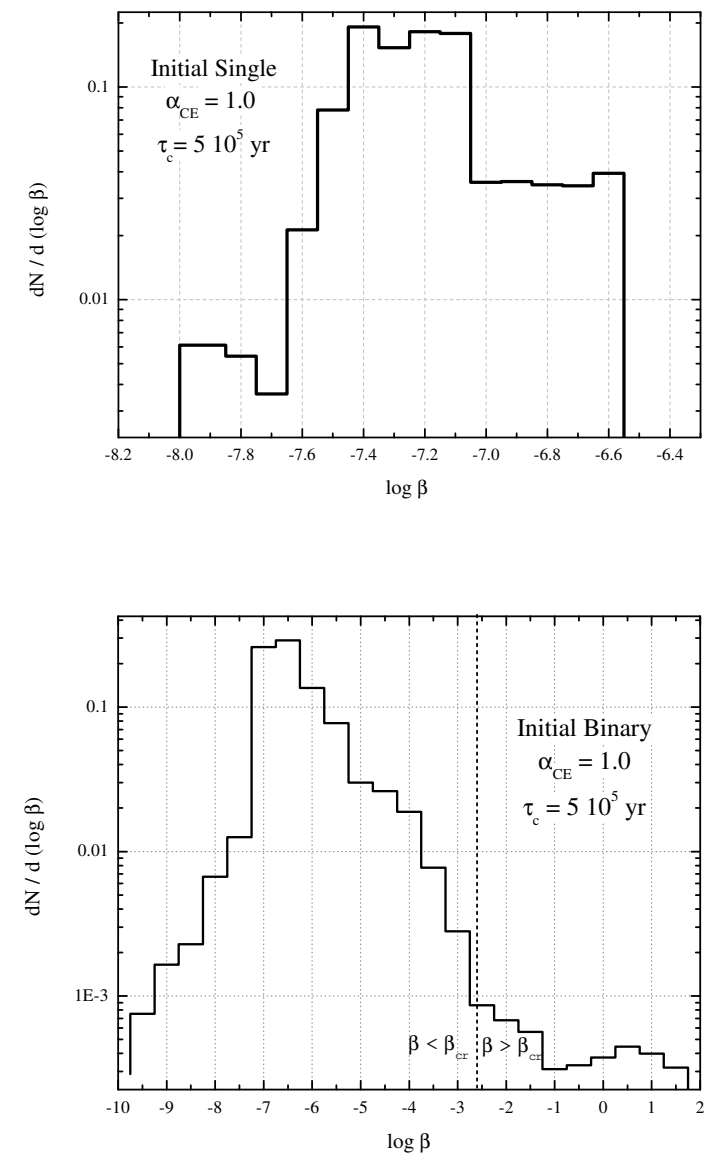

Figure 2. Distribution of the pre-collapse core rotation parameter $\beta=$ $T /|W|$ for single and binary NS progenitors.

lope stage (Lorenzo et al. 2016). If merging of two stellar cores occurs inside the common envelope, the angular momentum of the coalesced core, $J_{c}^{\prime}$, is set to be equal to the orbital angular momentum of the two contacting cores prior to the coalescence, $J_{c}^{\prime}=M_{c, 1} M_{c, 2} \sqrt{G\left(R_{c, 1}+R_{c, 2}\right) /\left(M_{c, 1}+M_{c, 2}\right)}$, where $M_{c, 1}, M_{c, 2}$ and $R_{c, 1}, R_{c, 2}$ are masses and radii of the cores, respectively. The rigid initial rotation and no coupling with the ejecting envelope is assumed.Most binary models that lead to the formation of rapidly rotating pre-SN cores start with mass ratio $q \sim 1$ (see Tables B1-B5 in Appendix B). Further rotational evolution of the newly formed helium core is treated in the same way as for ZAMS stars described above (i.e. the core-envelope structure with effective coupling $\tau_{c}$ is assumed).

The fraction of the rapidly rotating pre-collapse iron cores with $\beta>\beta_{c r} \sim 2.7 \times 10^{-3}$ among all core collapses are shown in Fig. 3 as a function of the CE efficiency parameter $\alpha_{C E}$ for three effective core-envelope coupling times $\tau_{c}=10^{5}, 5 \times 10^{5}$ years and $10^{6}$ years and for different formation channels listed in Tables B1B5. The second parameter describing the common envelope stage in the BSE code, the $\lambda$-parameter, which is the measure of the gravitational binding energy of the envelope, was calculated in all cases according to the BSE code prescriptions (Hurley et al. 2000). It is seen that depending on the parameters, in particular evolutionary channel this fraction varies by about two order of magnitude from 

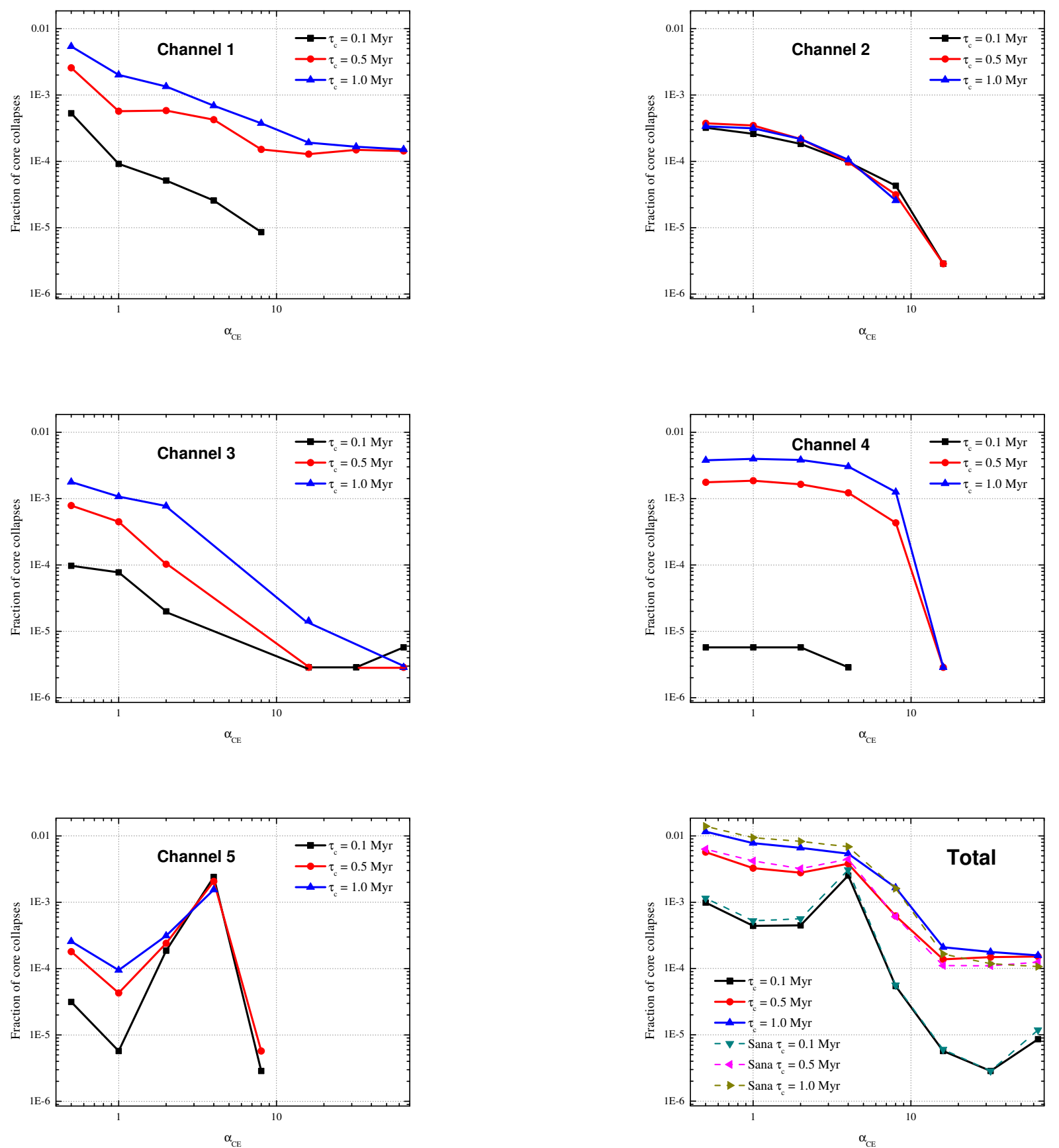

Figure 3. Fraction of rapidly rotating cores as a function of the common envelope parameter $\alpha_{C E}$ in different formation channels (Tables B1-B5) for three values of the core-envelope effective coupling time $\tau_{c}=0.1,0.5,1$ Myr. Bottom right panel shows the sum from all channels leading to core collapses for two initial binary orbital period distribution - the flat distribution (solid lines) and Sana's distribution (Sana et al. 2012).

a few $\times 10^{-5}$ to a few $\times 10^{-3}$, with larger fraction for more effective CE (i.e., with smaller $\alpha_{C E}$-parameter). The non-monotonic dependence of the formation rate in channels 3 and 5 on the CE efficiency parameter is due to the occurrence of several CE stages. Note that not all formation channels are possible at particular values of $\alpha_{C E}$. It is also seen that generally, with increasing $\tau_{c}$ the fraction of rapidly rotating cores increases as expected. Due to the weakening of the core-envelope angular momentum coupling, the envelope, which mainly expands and looses the momentum in stellar winds, does not effectively brake the stellar core. The total formation rate of rapidly rotating cores from all evolutionary channels (see the bottom right panel in Fig. 3) can be $10^{-3}-10^{-2}$ of all core collapses. The assumed initial mass ratio distribution of the binary components does not alter this result. 


\section{SUMMARY AND CONCLUSIONS}

In the present study, using the population synthesis method based on the BSE code, we calculated the expected fraction of the rapidly rotating pre-collapse stellar cores, which potentially can lead to the formation of dynamically unstable proto NSs. The critical rotation of the pre-collapse cores is quantified by the ratio of the rotational to gravitational energy, $\beta=T /|W|$. Potentially dynamically unstable proto-NS arises from cores with $\beta>\beta_{c r} \sim 2.7 \times 10^{-3}$. The collapse of such a rapidly rotating cores can result in fission of the core in two parts with the formation of binary proto-NS. Coalescences of such close binary NSs can be potential gravitational wave sources. To calculate the formation rate of such rapidly rotating stellar cores, the treatment of the core rotation in two-zone approximation was used. In this approach, the stellar core is assumed to be coupled with the envelope by Eq. (1), which has one free parameter - the effective coupling time $\tau_{c}$. The applicability of this simplified treatment of stellar core rotation was checked by direct calculation of evolution of a rotating $15 M_{\odot}$ star by MESA code (Appendix A).

To fix the core-envelope coupling time, we have calculated the expected distribution of initial spin periods of NSs formed from single and binary star evolution and compared it with the spin distribution of young NSs as inferred from pulsar statistical studies (Popov \& Turolla 2012). We have found that $\tau_{c}=5 \times 10^{5}$ years gives reasonable initial NS spin period distribution centered at $\sim 0.1 \mathrm{~s}$. Then the BSE population synthesis code supplemented with the core-envelope rotational coupling treatment by Eq. (1) was used to calculate the expected distribution of the core rotation parameter $\beta$ prior to the collapse (Fig. 2). It was found that rapidly rotating core with $\beta>2.7 \times 10^{-3}$ may arise from binary evolution. The tail of rapidly rotating cores appears as a result of mergings of two non-degenerate stellar helium cores during common envelope stages in several evolutionary channels (Tables B1-B5 in Appendix $\mathrm{B})$. The angular momentum of the merged core is mostly determined by the orbital motion of the stellar cores in the common envelope, and hence is insensitive to the assumed initial distribution of stellar rotation at the ZAMS stage. At a fixed $\tau_{c}$, the common envelope efficiency parameter $\alpha_{C E}$ mostly affects the formation rate of rapidly rotating cores. We have found that depending on $\alpha_{C E}$, the fraction of rapidly rotating cores among all core collapses for all possible evolutionary channels can reach $0.1-1 \%$, which can be interesting from the point of view of the formation of double protoNS from core collapses as possible gravitational wave sources.

Clearly, our calculations cannot definitely predict the formation rate of binary proto-NS, and should be considered only as upper limits. Future 3D calculations of the collapse of rapidly rotating stellar cores (K. Manukovsky et al., in progress) should clarify the fate of the collapse and the possibility of the formation of binary proto-NS through this evolutionary channel.

\section{ACKNOWLEDGEMENTS}

The authors acknowledge S.I. Blinnikov and K.V. Manukovsky for discussions and Dr. Thomas Tauris for critical remarks. The work of KP and NP (study of stellar rotation, writing the paper, analysis of the results) is supported by RSF grant 14-12-00203. The work of AK, DK and SP (numerical simulations, checking with MESA code, analysis of the initial NS spin periods) is supported by RFBR grant 14-02-00567. The authors acknowledge support from M.V. Lomonosov Moscow State University Program of Development.

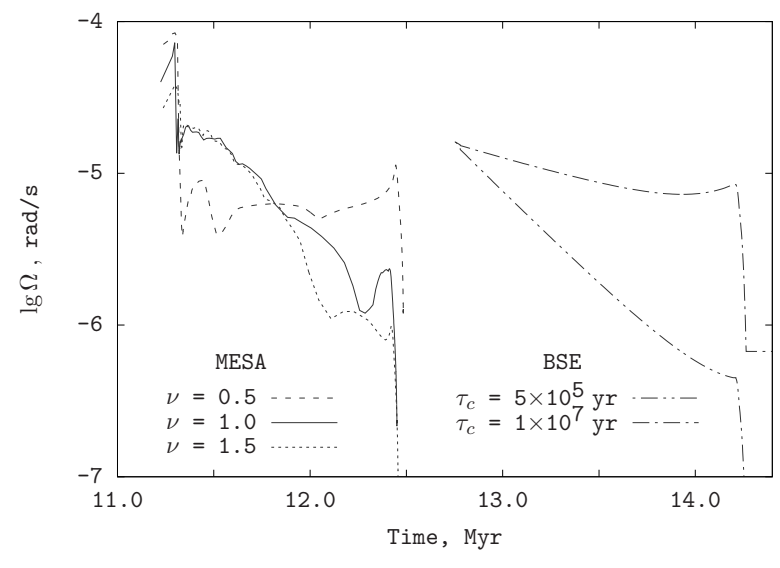

Figure 4. The comparison of the time evolution of the angular rotational velocity of the helium core of a $15 M_{\odot}$ star with initial rigid rotation calculated by MESA code (left curves) and BSE (right curves) for different values of the core-envelope coupling time $\tau_{c}$.

\section{APPENDIX A: COMPARISON WITH MESA CODE}

We have compared our prescription of the rotational core evolution of normal stars (Section 2) with calculations carried out by MESA code (Paxton et al. 2015). As an example, we have considered evolution of a $15 M_{\odot}$ star with solar metallicity and initial rotation velocity given by Eq. (107) of Hurley et al. (2000).

In the MESA code, implementation of rotation closely follows papers Pinsonneault et al. (1989); Heger et al. (2000, 2005) where the angular momentum transport formulates as a diffusive process. There are three adjustable efficiency factors to calibrate diffusion coefficients. The first parameter is the ratio of the turbulent viscosity to the diffusion coefficient, $f_{c}=D / v$. It changes the time scale of the chemical composition transport. The second parameter is $f_{\mu}$ which describes the sensitivity of the rotationally induced mixing to chemical composition $\mu$-gradients. The last parameter is the diffusion coefficient of the angular momentum $f_{\omega}$ which changes the time scale of the angular momentum transport. Following Heger et al. (2000), we set $f_{c}=1 / 30$ and $f_{\mu}=0.05$. Influence of the parameters $f_{\omega}$ on the models is not significant in our case and we set it equal to unity.

In the MESA code there are several other parameters related to different mechanisms responsible for the angular momentum transport across the stellar radius. In Table A1 we list the parameters and their values we have used in our calculations (http://mesa. sourceforge.net/).

We use the initial settings in the MESA version 8118 code at the star/test_suite/massive_rotating directory for Solar chemical composition, the variable mesh_delta_coeff was set to 0.8 for model convergence. The kinematic shear viscosity most significantly affects the angular momentum transport, and therefore we use three values of this parameter for three different models.

Fig. 4 shows the time evolution of the angular rotational velocity $\Omega$ of the He core of a $15 M_{\odot}$ star calculated by MESA (left curves) with different kinematic shear viscosity factor am_nu_visc_factor and by BSE (right curves) with different core-envelope coupling time $\tau_{c}$. The curves start at the time corresponding to the appearance of the He core after the main sequence. At the zero main sequence, the star is assumed to be rigidly rotating with the angular velocity calculated from Eq. (107) in Hurley et al. 
Table A1. Parameters in the MESA code used in model calculations of $\mathrm{He}$ core rotation of a $15 M_{\odot}$ star.

\begin{tabular}{lcc}
\hline \hline MESA parameter & Value & Comment \\
\hline D_DSI_factor & 1.0 & dynamical shear instability \\
D_SH_factor & 1.0 & Solberg-Hoiland parameter \\
D_SSI_factor & 1.0 & secular shear instability \\
D_ES_factor & 1.0 & Eddington-Sweet circulation \\
D_GSF_factor & 1.0 & Goldreich-Schubert-Fricke parameter \\
D_ST_factor & 1.0 & Spruit-Tayler dynamo \\
& & \\
am_nu_visc_factor & $0.5,1.0,1.5$ & kinematic shear viscosity $v$ \\
& & $f_{c}$ \\
am_D_mix_factor & $1 / 30$ & $f_{\omega}$ \\
am_nu_factor & 1.0 & $f_{\mu}$ \\
am_gradmu_factor & 0.05 & \\
\hline
\end{tabular}

(2000). The time shift between the MESA (left curves) and BSE (right curves) calculations is apparently due to different treatment of convection in the stellar evolution used in MESA and BSE. It is seen from Fig. 4 that the evolution of the rotational velocity of the stellar core with time calculated by MESA can reproduce much more simplified treatment using the effective core-envelope coupling, as described in Section 2 and implemented in the BSE code, by choosing the appropriate value of the kinematic viscosity coefficient $v$ in the MESA code. Thus we conclude that the use of the core-envelope coupling parameter $\tau_{c}$ in the BSE models with $\tau_{c}=5 \times 10^{5}-10^{6}$ yrs is in agreement with detailed MESA calculations.

\section{APPENDIX B: TYPICAL EVOLUTIONARY CHANNELS LEADING TO RAPIDLY ROTATING STELLAR CORES}

Here we provide examples of typical evolutionary tracks of binary stars that lead to the formation of rapidly rotating iron cores before collapse. Tracks are grouped in 'channels' according to the evolutionary stage of the components before the formation of the rotating core in the common envelope stage (CE). The designation of the evolutionary stages is the same as in the BSE code (MS - main sequence star, HG - post-MS star in the Hertzsprung gap, GB - star at the giant branch, $\mathrm{CHeB}$ - core helium burning, EAGB - early asymptotic giant branch, TPAGB - thermally pulsing AGB, HeMS - naked helium main sequence, HeHG - helium star in the Hertzsprung gap). Columns in the tables show: time in million years $(\mathrm{T})$, evolutionary stages of the components, masses of the components in solar masses (M1, M2), orbital separation in solar radii (A), radii of the components in units of the effective Roche lobe radius (R1/RL1, R2/RL2). 
Table B1. Channel 1: First Giant Branch + MS

\begin{tabular}{lccccccc}
\hline $\mathrm{T}$ & Star1 & Star2 & M1 & M2 & A & R1/RL1 & R2/RL2 \\
\hline 0.0 & MS & MS & 8.6 & 8.4 & 203 & 0.05 & 0.05 \\
32.4 & HG & MS & 8.5 & 8.3 & 204 & 0.11 & 0.11 \\
32.5 & HG & MS & 8.5 & 8.3 & 205 & 1.05 & 0.12 \\
32.5 & GB & MS & 8.5 & 8.4 & 201 & CE1 & - \\
32.5 & GB & - & 11.9 & - & - & - & - \\
32.5 & CHeB & - & 11.9 & - & - & - & - \\
36.3 & EAGB & - & 11.6 & - & - & - & - \\
\multicolumn{7}{l}{ Core collapse } \\
\hline \hline
\end{tabular}

Table B2. Channel 2: Core He-burning + shell He-burning

\begin{tabular}{|c|c|c|c|c|c|c|c|}
\hline $\mathrm{T}$ & Star1 & Star2 & M1 & M2 & A & R1/RL1 & R2/RL2 \\
\hline 0.0 & MS & MS & 10.3 & 10.0 & 1147 & 0.01 & 0.01 \\
\hline 23.4 & HG & MS & 10.1 & 10.0 & 1159 & 0.02 & 0.02 \\
\hline 23.5 & GB & MS & 10.1 & 10.0 & 1160 & 0.45 & 0.02 \\
\hline 23.5 & $\mathrm{CHeB}$ & MS & 10.1 & 10.0 & 1103 & 0.96 & 0.03 \\
\hline 24.2 & $\mathrm{CHeB}$ & HG & 9.9 & 10.0 & 1106 & 0.66 & 0.02 \\
\hline 24.3 & $\mathrm{CHeB}$ & GB & 9.9 & 10.0 & 1106 & 0.65 & 0.46 \\
\hline 26.2 & EAGB & $\mathrm{CHeB}$ & 9.7 & 9.6 & 1064 & 0.97 & 0.12 \\
\hline 26.2 & EAGB & $\mathrm{CHeB}$ & 9.7 & 9.6 & 1031 & 1.02 & 0.13 \\
\hline 26.2 & EAGB & CHeB & 9.7 & 9.6 & 1031 & CE1 & - \\
\hline 26.2 & $\mathrm{CHeB}$ & - & 16.6 & - & - & & - \\
\hline \multirow[t]{2}{*}{26.4} & EAGB & - & 15.0 & - & - & - & - \\
\hline & \multicolumn{7}{|c|}{ Core collapse } \\
\hline
\end{tabular}

Table B3. Channel 3: naked helium main sequence + shell H-burning

\begin{tabular}{|c|c|c|c|c|c|c|c|}
\hline $\mathrm{T}$ & Star1 & Star2 & M1 & M2 & A & R1/RL1 & R2/RL2 \\
\hline 0.0 & MS & MS & 10.1 & 9.3 & 390 & 0.03 & 0.03 \\
\hline 23.8 & HG & MS & 10.0 & 9.3 & 393 & 0.06 & 0.05 \\
\hline 23.9 & HG & MS & 10.0 & 9.3 & 394 & 1.03 & 0.05 \\
\hline 23.9 & GB & MS & 10.0 & 9.3 & 381 & CE1 & - \\
\hline 27.7 & HeMS & HG & 2.1 & 9.2 & 52.5 & 0.03 & 0.33 \\
\hline 27.7 & HeMS & HG & 2.1 & 9.2 & 52.7 & 0.03 & 1.00 \\
\hline 27.7 & HeMS & HG & 2.1 & 9.2 & 52.7 & - & CE2 \\
\hline 27.7 & - & $\mathrm{CHeB}$ & - & 8.1 & - & - & - \\
\hline 29.9 & - & EAGB & - & 8.0 & - & - & - \\
\hline 30.0 & - & TPAGB & - & 7.9 & - & - & - \\
\hline & \multicolumn{7}{|c|}{ Core collapse } \\
\hline
\end{tabular}

Table B4. Channel 4: naked helium sub giant + shell H-burning

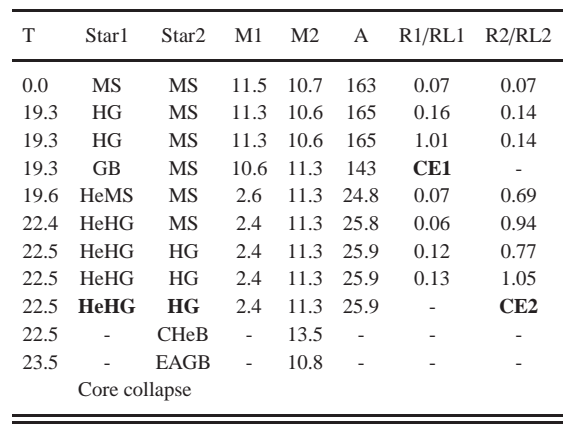

\section{REFERENCES}

Aasi J., et al., 2015, Classical and Quantum Gravity, 32, 115012 Abadie J., et al., 2010, Classical and Quantum Gravity, 27, 173001 Abbott B. P., et al., 2016, preprint, (arXiv: 1605.01785)

Acernese F., et al., 2015, Classical and Quantum Gravity, 32, 024001 Allain S., 1998, A\&A, 333, 629

Ardeljan N. V., Bisnovatyi-Kogan G. S., Moiseenko S. G., 2005, MNRAS, 359,333
Table B5. Channel 5: naked helium sub giant + naked helium sub giant

\begin{tabular}{|c|c|c|c|c|c|c|c|}
\hline $\mathrm{T}$ & Star1 & Star2 & M1 & M2 & A & R1/RL1 & R2/RL2 \\
\hline 0.0 & MS & MS & 12.5 & 12.5 & 1175.0 & 0.01 & 0.01 \\
\hline 16.7 & HG & MS & 12.3 & 12.3 & 1194.4 & 0.02 & 0.03 \\
\hline 16.7 & GB & MS & 12.3 & 12.3 & 1194.8 & 0.63 & 0.03 \\
\hline 16.7 & GB & MS & 12.3 & 12.3 & 1140.4 & 1.00 & 0.03 \\
\hline 16.7 & GB & MS & 12.3 & 12.3 & 1140.4 & CE1 & - \\
\hline 16.7 & HeMS & MS & 2.9 & 12.3 & 202.2 & 0.01 & 0.12 \\
\hline 16.7 & HeMS & HG & 2.9 & 12.3 & 202.2 & 0.01 & 0.11 \\
\hline 16.8 & HeMS & HG & 2.9 & 12.3 & 202.3 & 0.01 & 1.05 \\
\hline 16.8 & HeMS & HG & 2.9 & 12.3 & 202.3 & - & CE2 \\
\hline 16.8 & HeMS & HeMS & 2.9 & 2.9 & 1.8 & 0.63 & 0.62 \\
\hline 19.3 & HeHG & $\mathrm{HeHG}$ & 2.7 & 2.7 & 1.6 & 1.00 & 0.68 \\
\hline 19.3 & HeHG & HeHG & 2.7 & 2.7 & 1.6 & CE3 & - \\
\hline \multirow[t]{2}{*}{19.3} & HeHG & - & 5.1 & - & - & - & - \\
\hline & \multicolumn{7}{|c|}{ Core collapse } \\
\hline
\end{tabular}

Baiotti L., de Pietri R., Manca G. M., Rezzolla L., 2007, Phys. Rev. D, 75,044023

Bisnovatyi-Kogan G. S., 1971, Sov. Astron., 14, 652

Blanchet L., 2014, Living Reviews in Relativity, 17

Bogomazov A. I., Popov S. B., 2009, Astronomy Reports, 53, 325

Braithwaite J., Spruit H. C., 2015, preprint, (arXiv: 1510.03198)

Centrella J. M., New K. C. B., Lowe L. L., Brown J. D., 2001, ApJL, 550, L193

Chandrasekhar S., 1969, Ellipsoidal figures of equilibrium

Clark D. H., Stephenson F. R., 1982, in Rees M. J., Stoneham R. J., eds, NATO Advanced Science Institutes (ASI) Series C Vol. 90, NATO Advanced Science Institutes (ASI) Series C. pp 355-370

Clark J. S., Ritchie B. W., Najarro F., Langer N., Negueruela I., 2014, A\&A, 565, A90

Colgate S. A., White R. H., 1966, ApJ, 143, 626

Colpi M., Wasserman I., 2002, ApJ, 581, 1271

Davies M. B., King A., Rosswog S., Wynn G., 2002, ApJL, 579, L63

Davies B., Figer D. F., Kudritzki R.-P., Trombley C., Kouveliotou C., Wachter S., 2009, ApJ, 707, 844

Durisen R. H., Tohline J. E., 1985, in Black D. C., Matthews M. S., eds, Protostars and Planets II. pp 534-575

Faucher-Giguère C.-A., Kaspi V. M., 2006, ApJ, 643, 332

Fuller J., Cantiello M., Lecoanet D., Quataert E., 2015, preprint, (arXiv: 1502.07779)

Gossan S. E., Sutton P., Stuver A., Zanolin M., Gill K., Ott C. D., 2016, Phys. Rev. D, 93, 042002

Guilet J., Fernández R., 2014, MNRAS, 441, 2782

Gullón M., Miralles J. A., Viganò D., Pons J. A., 2014, MNRAS, 443, 1891

Gullón M., Pons J. A., Miralles J. A., Viganò D., Rea N., Perna R., 2015, preprint, (arXiv: 1507.05452)

Hayama K., Kuroda T., Nakamura K., Yamada S., 2016, Physical Review Letters, 116, 151102

Heger A., Langer N., Woosley S. E., 2000, ApJ, 528, 368

Heger A., Woosley S. E., Spruit H. C., 2005, ApJ, 626, 350

Hurley J. R., Pols O. R., Tout C. A., 2000, MNRAS, 315, 543

Hurley J. R., Tout C. A., Pols O. R., 2002, MNRAS, 329, 897

Igoshev A. P., Popov S. B., 2013, MNRAS, 432, 967

Imshennik V. S., 1992, Soviet Astronomy Letters, 18, 194

Imshennik V. S., 2000, Physics Uspekhi, 43, 509

Imshennik V. S., 2008, Astronomy Letters, 34, 375

Imshennik V. S., 2011, Physics Uspekhi, 53, 1081

Imshennik V. S., Molokanov V. O., 2009, Astronomy Letters, 35, 799

Imshennik V. S., Nadyozhin D. K., 1992, Soviet Astronomy Letters, 18, 79

Imshennik V. S., Popov D. V., 1998, Astronomy Letters, 24, 206

Langer N., 2012, ARAA, 50, 107

LeBlanc J. M., Wilson J. R., 1970, ApJ, 161, 541

Lipunova G. V., Gorbovskoy E. S., Bogomazov A. I., Lipunov V. M., 2009, MNRAS, 397, 1695

Lorenzo J., Negueruela I., Vilardell F., Simón-Díaz S., Pastor P., Méndez Majuelos M., 2016, preprint, (arXiv: 1603.03177) 


\section{Postnov et al.}

Maeder A., Meynet G., 2000, ARAA, 38, 143

Melson T., Janka H.-T., Bollig R., Hanke F., Marek A., Müller B., 2015, ApJL, 808, L42

Menou K., Perna R., Hernquist L., 2001, ApJL, 554, L63

Miller M. C., Miller J. M., 2015, Phys. Rep., 548, 1

Mösta P., Ott C. D., Radice D., Roberts L. F., Schnetter E., Haas R., 2015, preprint, (arXiv: 1512.00838)

Muno M. P., et al., 2006, ApJL, 636, L41

Noutsos A., Schnitzeler D. H. F. M., Keane E. F., Kramer M., Johnston S., 2013, MNRAS, 430, 2281

Paxton B., Bildsten L., Dotter A., Herwig F., Lesaffre P., Timmes F., 2011, ApJ Suppl. Ser., 192, 3

Paxton B., et al., 2015, ApJ Suppl. Ser., 220, 15

Pinsonneault M. H., Kawaler S. D., Sofia S., Demarque P., 1989, ApJ, 338,424

Popov S. B., Prokhorov M. E., 2006, MNRAS, 367, 732

Popov S. B., Turolla R., 2012, Astroph.Sp.Sci., 341, 457

Popov S. B., Pons J. A., Miralles J. A., Boldin P. A., Posselt B., 2010, MNRAS, 401, 2675

Postnov K. A., Yungelson L. R., 2014, Living Reviews in Relativity, 17

Sana H., et al., 2012, Science, 337, 444

Sathyaprakash B., Schutz B. F., 2009, Living Reviews in Relativity, 12

Spruit H. C., 2002, A\&A, 381, 923

Spruit H., Phinney E. S., 1998, Nature, 393, 139

Thompson C., Duncan R. C., 1993, ApJ, 408, 194

Turolla R., Zane S., Watts A. L., 2015, Reports on Progress in Physics, 78,116901

Vivekanand M., Narayan R., 1981, Journal of Astrophysics and Astronomy, 2,315

Voss R., Tauris T. M., 2003, MNRAS, 342, 1169

Vranesevic N., et al., 2004, ApJL, 617, L139

Zahn J.-P., Talon S., Matias J., 1997, A\&A, 322, 320

de Mink S. E., Belczynski K., 2015, ApJ, 814, 58 